Cheng, J.C.P., Chen, K. and Chen, W. (2017). "Comparison of marker-based AR and markerless AR: A case study on indoor decoration system." In: LC3 2017: Volume I - Proceedings of the Joint Conference on Computing in Construction (JC3), July 4-7, 2017, Heraklion, Greece, pp. 483-490. DOI: https://doi.org/10.24928/JC3-2017/0231.

\title{
COMPARISON OF MARKER-BASED AR AND MARKERLESS AR: A CASE STUDY ON INDOOR DECORATION SYSTEM
}

\author{
Jack C.P. Cheng ${ }^{1}$, Keyu Chen ${ }^{2}$, and Weiwei $\mathrm{Chen}^{3}$
}

\begin{abstract}
The feature of combining the real world with virtual objects enables Augmented Reality (AR) to provide a better display of information, resulting in its increasing popularity in a variety of industries. However, sometimes the two types of AR application - marker-based $\mathrm{AR}$ and markerless AR - are confusing to developers. These two types of AR can be applied to different situations because both of them have their own advantages and disadvantages. So it is necessary for developers to learn the difference between these two types of AR. In this paper, an AR-based indoor decoration system is developed to illustrate the difference between markerbased AR and markerless AR. The developed system can aid in viewing the effect of indoor decoration with both virtual facilities and product information in advance. Based on the two modules of this system (marker-based module and markerless module), this paper makes a comparison between these two types of AR in the following four aspects: (1) methods in realizing AR, (2) position accuracy, (3) stability, and (4) hardware support. The comparison results can provide future developers with a judgement basis when deciding which types of AR to select.
\end{abstract}

Keywords: Marker-based AR, markerless AR, indoor decoration, comparison.

\section{INTRODUCTION}

Augmented Reality (AR) is an innovative human-machine interaction technology that enables digital information such as virtual objects, animations, and images to be overlaid on the real world to achieve a natural interaction with users. Compared with Virtual Reality (VR), which allows users to be immersed in a complete virtual environment, this seamless combination technology uses computational methods to combine the virtual world and real world, thus information of the users' surrounding environment becomes readable and manipulable. Through prototyping and visualization of products, many of the largest companies in the world have been using AR to enable consumers to experience products in novel ways (Scholz and Smith 2016).

Generally, there are two types of AR - one is called marker-based AR, the other is called markerless AR. Currently, no official definitions of marker-based AR and markerless AR are available. But from their literal meaning it can be implied that marker-based AR has to use a marker as a trigger while markerless AR can be used without markers. For marker-based AR, marker can be either a 2D image with visual features that are easy to be extracted (Lepetit and Fua, 2005) or natural objects directly in the real environment (Feng and Kamat 2012). Instead of tracking features of a marker, markerless AR usually uses some types of localization technology, such as Global Positioning System (GPS) (Behzadan et al. 2008), Radio Frequency Identification (RFID) (Beneš et

1 Associate Professor, Department of Civil and Environmental Engineering, The Hong Kong University of Science and Technology, Hong Kong, cejcheng@ust.hk

2 Ph.D Student, Department of Civil and Environmental Engineering, The Hong Kong University of Science and Technology, Hong Kong, kchenal@connect.ust.hk

3 Ph.D Student, Department of Civil and Environmental Engineering, The Hong Kong University of Science and Technology, Hong Kong, wchenau@connect.ust.hk 
al. 2013), and sensor technology (Gerstweiler et al. 2015) to control the relative position relationship between virtual objects and the real world.

As the AEC industry has long been regarded as a highly resource consuming industry, innovations in this industry are of vital importance. Since Caudell (1995) used the term "Augmented Reality" for the first time, AR has been increasingly applied to different fields, and the AEC industry is no exception. Through combining virtual objects with the real world, AR technology can aid in architectural design (Altabtabai and Yan 2015), construction management (Rohani et al. 2013), facility management (Hou et al. 2013), etc. To decide whether to use marker-based AR or markerless $\mathrm{AR}$, it is necessary for developers to learn the difference between these two types of AR technology, especially the technology behind them. However, the existing AR applications in the AEC industry are either marker-based or markerless - none of them have both modules at the same time. It is difficult to make a comparison between these two types if the application has only one module. In 2015, a comparison between marker-based AR and markerless AR has been done by Stoyanova et al. (2015). The research team developed an AR shopping platform with both marker-based version and markerless version and a questionnaire was conducted to evaluate the performance of both versions respectively. However, this analytical survey focused on user experience only because the results of comparison totally came from users' perspective. For developers, it is more important to differentiate marker-based AR with markerless AR in terms of technology, rather than user experience.

Therefore, an AR-based indoor decoration system is developed in this study, based on which marker-based $\mathrm{AR}$ and markerless $\mathrm{AR}$ are compared in terms of technology from the perspectives of system developers. The function of the developed system is to aid in viewing the effect of indoor decoration with both virtual facilities and product information in advance. Based on the two modules of this system (marker-based module and markerless module), this paper makes a general comparison between these two types of AR in the following aspects: (1) methods in realizing AR, (2) position accuracy, (3) stability, and (4) hardware support. The comparison results can provide future developers with a judgement basis when deciding which types of AR to select.

\section{THE DEVELOPED AR-BASED INDOOR DECORATION SYSTEM}

\subsection{Features of the Developed AR System}

The function of the developed AR-based indoor decoration system is to superimpose a virtual facility or furniture onto a particular location in the real indoor environment. The combination of virtual products with the real environment can help users preview the indoor decoration effect before purchasing the real products. As shown in Figure 1, a lamp is displayed on a partition with corresponding information shown in the lower right corner of the screen. Users can select different products using the dropdown list on the left side of the screen. The products can be moved, scaled or rotated by clicking the mouse of a desktop computer or touch the screen of a mobile device. 


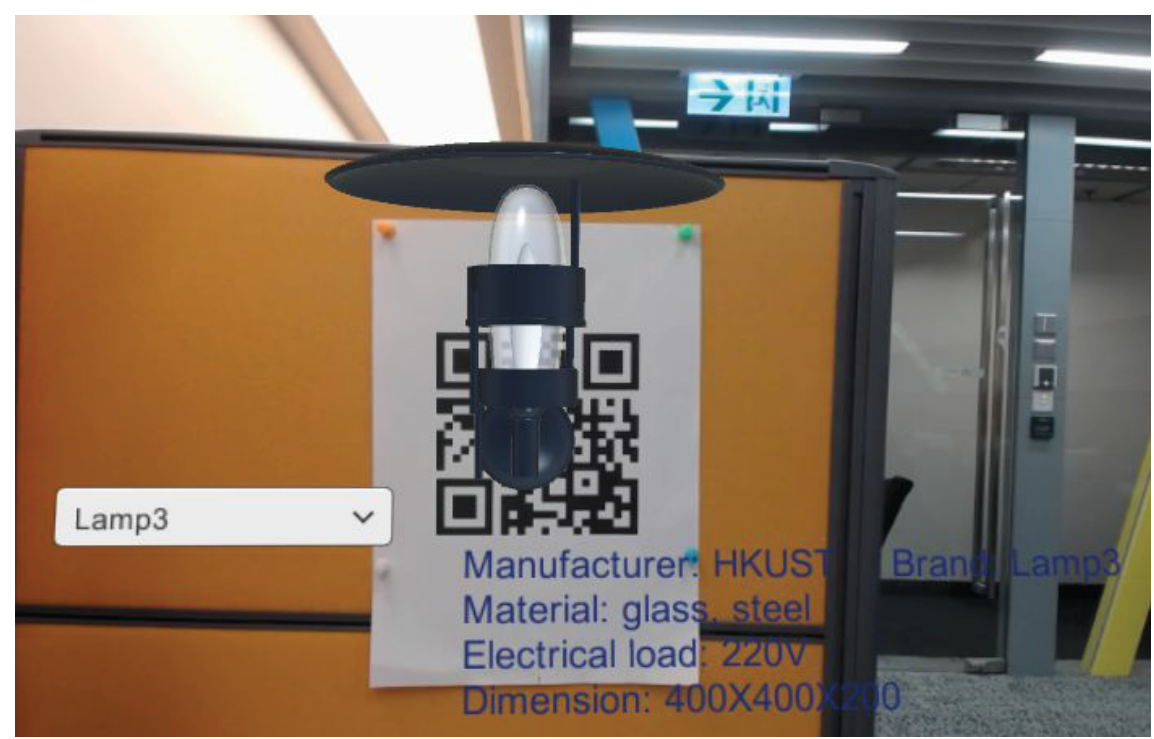

Figure 1: The developed AR-based indoor decoration system

The objective of this study is to figure out the difference between marker-based AR and markerless AR. Therefore, an important requirement for the developed AR system is that the system should support both marker-based AR and markerless AR at the same time. For the marker-based module, users need to put a marker somewhere in the room and the products will appear after scanning the marker. For the markerless module, users can view the products if their locations are close enough to the virtual address of the products.

\subsection{Development of the Marker-based Module}

A commonly used AR Software Development Kit (SDK) is selected to build the marker-based module of the decoration system. In the development environment, each virtual product is linked with a particular marker. The algorithms in the AR SDK can extract geometric features from a given marker and match the corresponding product. Then the marker serves as a spatial reference to display the corresponding product on the screen.

\subsection{Development of the Markerless Module}

The markerless module is based on GPS technology. In the development environment, each virtual product is assigned a coordinate value of longitude and latitude. When the system is installed on a mobile device, it can get the current location using the GPS function of the device. If the difference of longitude and latitude value between the device and the virtual product is less than 0.01 degree, the product will be displayed on the screen. Then the gyroscope is used to determine the relative angle relationship and the camera is used to turn the display background into the real environment.

\section{COMPARISON BETWEEN MARKER-BASED AR AND MARKERLESS AR}

Based on the developed indoor decoration system, this paper makes a comparison between markerbased AR and markerless AR. Table 1 shows the results of the comparison. 
Comparison of Marker-based AR and Markerless AR: A Case Study on Indoor Decoration System

Table 1: Comparison between marker-based AR and markerless AR

\begin{tabular}{|c|c|c|c|}
\hline \multicolumn{2}{|c|}{ Comparison Aspects } & \multirow{2}{*}{$\begin{array}{c}\text { Marker-based AR } \\
\text { Depends on markers }\end{array}$} & \multirow{2}{*}{$\begin{array}{c}\text { Markerless AR } \\
\begin{array}{c}\text { Depends on localization technology } \\
\text { and gyroscope }\end{array}\end{array}$} \\
\hline & $\begin{array}{c}\text { Relative } \\
\text { position/angle }\end{array}$ & & \\
\hline Methods & $\begin{array}{l}\text { AR Software } \\
\text { Development } \\
\text { Kit (SDK) }\end{array}$ & Commonly used & Rarely used \\
\hline \multirow{2}{*}{$\begin{array}{l}\text { Position } \\
\text { accuracy }\end{array}$} & High/low & Relatively higher & Relatively lower \\
\hline & $\begin{array}{l}\text { Influential } \\
\text { factors }\end{array}$ & Brightness & Localization technology \\
\hline \multirow[b]{2}{*}{ Stability } & High/low & Relatively lower & Relatively higher \\
\hline & $\begin{array}{l}\text { Influential } \\
\text { factors }\end{array}$ & Markers and SDKs & $\begin{array}{l}\text { Localization technology and } \\
\text { gyroscopes }\end{array}$ \\
\hline \multirow{2}{*}{$\begin{array}{l}\text { Hardware } \\
\text { support }\end{array}$} & Desktop & Supported & Usually not supported \\
\hline & Mobile & supported & Supported \\
\hline
\end{tabular}

\subsection{Methods in Realizing AR}

The most obvious difference between marker-based AR and markerless AR is whether a marker is needed or not, which directly determines the way to set the relative position relationship between virtual objects and the real world.

To develop a marker-based AR application, the most important step is to identify a marker through the extracted features and superimpose virtual objects in the real environment. To identify a marker, particular algorithms are needed to extract features from the marker and recognize it. Currently there are several AR SDKs available in market, providing these kinds of algorithms for developers. The algorithms in the AR SDKs can automatically detect and extract features from a maker. After the virtual objects are linked to the marker, the relative position relationship can be set in the development environment (e.g. a 3D development engine or development scripts). The real position of the augmented objects can be adjusted by changing the relative position relationship among virtual AR camera (represents the screen where the augmented objects to be displayed), objects and markers in the development environment.

To develop a markerless AR application, usually some localization technologies are used to set the relative position relationship between virtual objects and users. Currently, most existing AR SDKs are developed for marker-based AR, while only few of them support markerless AR. As a result, developers normally select a certain type of localization technology and develop their own framework to form the relationship between virtual objects and the real world. In the markerless module of the developed indoor decoration system, GPS, a gyroscope and a camera are used to achieve the AR function. Each virtual objects are assigned a position, which is represented by latitude and longitude. Through the GPS function of the mobile device, the relative position relationship between the virtual objects and the device can be determined. Then a gyroscope on the mobile device is used to determine the relative angle relationship between the virtual objects and 
the device. At the same time, the camera of the device turns the displayed background on the screen into the real background.

In conclusion, developers can use existing AR SDKs to develop marker-based AR applications, while they usually have to develop their own framework based on localization technologies to develop markerless AR applications. Normally it has less complexity in developing marker-based AR applications because they do not have to write their own algorithms to extract features from markers.

\subsection{Position accuracy}

The position accuracy of marker-based AR applications is so high that we do not need to take it into account. It is because the augmented objects will be superimposed onto the marker just as it has been set in the development environment. For instance, in the developed indoor decoration system, after the QR code has been scanned, the products will be shown in front of the QR code. In this process, the tiny error in position is negligible.

On the other hand, the accuracy of markerless AR application is relatively lower. In the markerless module of the developed system, the recognition range is \pm 0.01 degree in latitude and longitude, but it does not represent the accuracy of this system. In fact, the accuracy of this system depends on the accuracy of its localization technology. According to the official U.S Government information about GPS, the URE (User Range Error) for civil commitments is 0.8 meters (NOAA, 2014). But the accuracy of the developed system is much lower when being tested on a common smart phone in indoor environment due to the GPS signal block by the building. For instance, the GPS localization result does not change while the user is walking within 10 meters, which means the accuracy of this system is lower than 10 meters. Under such circumstance, it is impossible to link the augmented products with any particular rooms.

Through the comparison of the marker-based module and the markerless module, it can be concluded that the position accuracy of marker-based AR applications is usually much higher than that of markerless AR applications. To improve the position accuracy of markerless AR, more accurate localization technologies such as those based on RFID, Wi-Fi, or Ultra-Wide Band (UWB) can be used to set the relative position relationship between virtual objects and the real world. For example, the accuracy of UWB localization system can be $1 \mathrm{~cm}$ (Tüchler et al 2005), which is even high enough for indoor AR applications.

\subsection{Stability}

Although the accuracy of marker-based AR applications is relatively higher compared with markerless AR applications, the stability of marker-based AR is not satisfying. Using current AR SDKs, the virtual objects are always shaky. In some cases, the augmented objects will even flash from time to time. Possible reasons include number of feature points of markers, complexity of virtual objects, AR SDKs, performance of hardware, darkness of the surrounding environment, etc. In order to verify some of the influential factors of stability for both marker-based and markerless AR applications, a series of controlled experiment are conducted as follows.

The first experiment was conducted to verify the influence of markers, as shown in Fig. 2. The two applications were using different markers - the first one used QR code which had around 1000 feature points to be extracted by the identifying algorithms, while the second one used some simple geometries as a marker which only had around 50 feature points to be recognized. All of the other conditions remained the same. The result showed that the augmented lamp in the second application shook more frequently with larger magnitude. Moreover, the lamp in the second application disappeared from time to time while moving the camera around the marker. The result 
of this experiment indicated that richness of markers was one of the factor that influenced the stability of marker-based AR applications. Besides complexity, the richness of feature points can also be influenced by the thickness of markers. Meanwhile, the further the distance between the marker and the camera, the greater the magnitude of shake would be. It was because when the marker was far from the camera, the feature points would only cover small proportion of the screen, leading to the difficulty of extracting features from the marker.
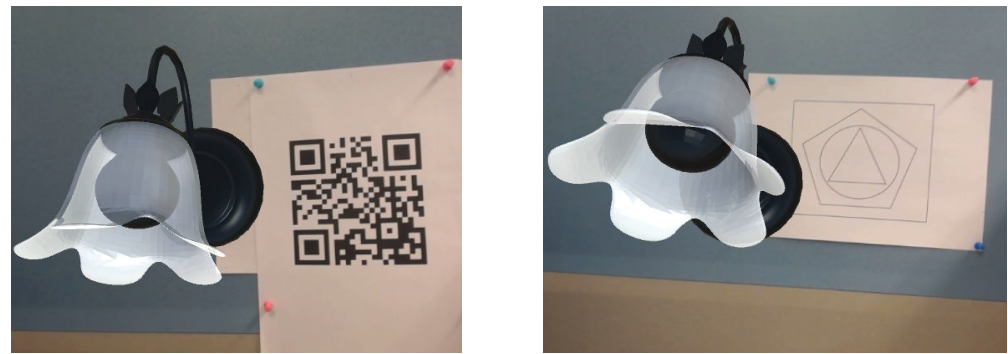

Figure 2. (Left) Result of marker-based AR using a QR code as the marker. (Right) Result of marker-based AR using simple geometries as the marker
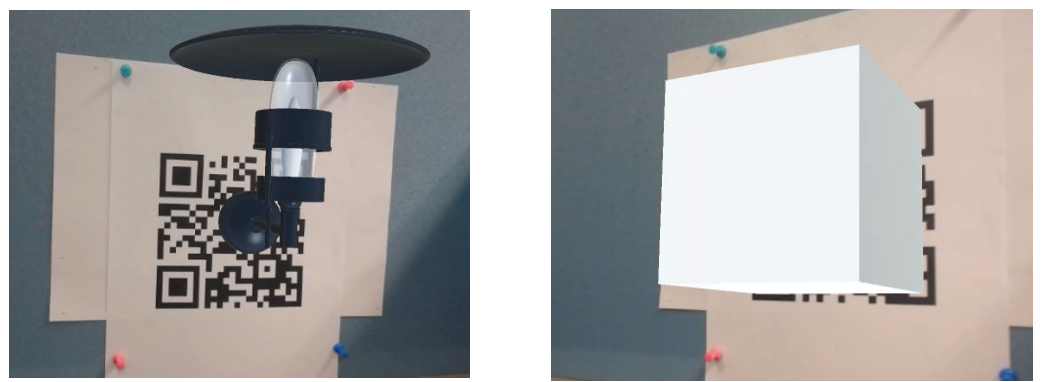

Figure 3. (Left) Result of marker-based AR augmenting a virtual lamp. (Right) Result of marker-based AR augmenting a virtual cube

The second experiment was conducted to verify the influence of complexity of the objects to be augmented, as shown in Fig. 3. The first application augmented a virtual lamp with around 13000 triangle faces while the second application augmented a virtual cube with only 12 triangle faces. All of the other conditions remained the same. However, the results showed that the stability of the second application was almost the same as the first one. It could be concluded that the complexity of models had little effect on the stability of AR applications.

The third experiment was conducted to verify the influence of AR SDKs, as shown in Fig. 4, because different algorithms had different efficiency. Two commonly used AR SDKs (one is renamed AR SDK A and the other is renamed AR SDK B) were selected as the samples of comparison. In the experiment, both AR SDKs used their own algorithms to extract features from the same marker and display a same model. After comparing, the stability of the application developed by using AR SDK A turned out to be much better. The result illustrated that maturity of AR SDKs had a direct impact on the stability of marker-based AR applications.

Although the stability of markerless AR is relatively higher than marker-based AR, there are still some factors that can influence the stability of markerless AR and make it unstable. The most obvious influential factor in this developed AR system is the stability of the used localization technology. Because of the unstability of indoor GPS signal, sometimes the positioning value suddenly changes even when the user does not move, leading to the suspension of AR function and disappearance of augmented objects. Besides the localization technology, gyroscope can also 
influence the stability of markerless AR application. When the developed system was being tested on a smart phone with a weak gyroscope, the augmented objects kept moving on the screen.
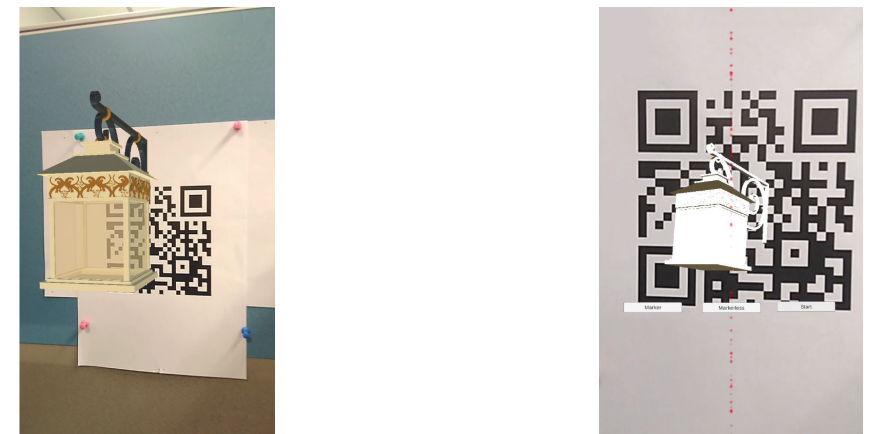

Figure 4: (Left) Result of marker-based AR using AR SDK A. (Right) Result of marker-based AR using AR SDK B.

In conclusion, the stability of marker-based AR is relatively lower, which is greatly influenced by the quality of markers and maturity of AR SDKs while the complexity of virtual models has little effect on it. To develop a stable marker-based AR application, developers need to select a proper AR SDK, build markers with high quality, and even optimize the existing feature recognition algorithms if necessary. For markerless AR, developers can use better localization technology and better hardware to improve the stability.

\subsection{Hardware Support}

Marker-based AR supports both desktop computers and mobile devices like smart phones. The marker-based module of the developed indoor decoration system has been tested on a desktop computer and a smart phone, both of which work fine with the system. For mobile devices like a commonly used smart phone, a laptop or a tablet, no extra equipment is needed because the device itself has already integrated all the required components. For a desktop computer, an extra camera must be connected to serve as a data input channel.

However, markerless AR is not supported in typical desktop computers, due to the lack of necessary components. For instance, in the case of the developed AR system, the AR function is based on gyroscope and GPS technology while a typical desktop computer has neither a GPS receiver nor a gyroscope. Although AR technology is more suitable for field applications, some AR applications are technically using desktop computers for design illustration purpose (Hou et al 2013). Meanwhile, to run the developed application on a mobile device, the device should have a GPS receiver and a gyroscope.

\section{CONCLUSIONS}

By comparing marker-based AR with markerless AR based on the developed decoration system, this paper makes the following conclusions: (1) the complexity in developing markerless AR is normally higher because the current existing AR SDKs support marker-based AR better; (2) the accuracy of marker-based AR is much higher while the accuracy of markerless AR can be improved by using more accurate localization technologies such as those based on RFID, Wi-Fi, or UWB; (3) the stability of marker-based AR is relatively lower, which is greatly influenced by the quality of markers and maturity of AR SDKs while the complexity of virtual models has little effect on it; and (4) marker-based AR is usually supported by both desktop computers and mobile devices while 
markerless AR is often supported by mobile devices only. The results of comparison can provide future developers with a judgement basis when deciding which types of AR to select.

\section{REFERENCES}

Altabtabai, J., \& Yan, W. (2015). A user interface for parametric architectural design reviews. Proceedings of the 20th Conference of the Association for Computer-Aided Architectural Design Research in Asia (CAADRIA), Daegu, Korea, 18-23 May, 2015.

Azuma, R., Baillot, Y., Behringer, R., Feiner, S., Julier, S., \& MacIntyre, B. (2001). Recent advances in augmented reality. IEEE Computer Graphics and Applications, 21(6), 34-47, doi: $10.1109 / 38.963459$.

Behzadan, A. H., Timm, B. W., \& Kamat, V. R. (2008). General-purpose modular hardware and software framework for mobile outdoor augmented reality applications in engineering. Advanced Engineering Informatics, 22(1), 90-105, doi.org/10.1016/j.aei.2007.08.005.

Beneš, F., Kubáĉ, L., Staša, P., Kebo, V., \& Kodym, O. (2013). The potential of RFID and augmented reality. Proceedings of the 13th International Multidisciplinary Scientific GeoConference SGEM, Albena, Bulgaria, 16th - 22nd June 2013.

Caudell, T. P. (1995). Introduction to augmented and virtual reality. Photonics for Industrial Applications (pp. 272-281), Boston, MA, 21 December 1995.

Feng, C., \& Kamat, V. R. (2012). Augmented reality markers as spatial indices for indoor mobile AECFM applications. Proceedings of 12th International Conference on Construction Applications of Virtual Reality (CONVR 2012), Taipei, Taiwan, 1-2 November 2012.

Gerstweiler, G., Vonach, E., \& Kaufmann, H. (2015). Hymotrack: A mobile AR navigation system for complex indoor environments. Sensors, 16(1), 17, doi:10.3390/s16010017.

Hou, L., Wang, X., \& Truijens, M. (2013). Using augmented reality to facilitate piping assembly: an experiment-based evaluation. Journal of Computing in Civil Engineering, 29(1), 05014007. doi: 10.1061/(ASCE)CP.1943-5487.0000344

Lepetit, V., \& Fua, P. (2005). Monocular model-based 3D tracking of rigid objects: A Survey, Foundations and Trends in Computer Graphics and Vision, 1(1), 1-89, doi:10.1561/0600000001

NOAA. (2014). GPS Accuracy. from http://www.gps.gov/systems/gps/performance/accuracy/

Rohani, M., Fan, M., \& Yu, C. (2013). Advanced visualization and simulation techniques for modern construction management. Indoor and Built Environment, 1420326X13498400, doi: $10.1177 / 1420326 X 13498400$.

Scholz, J., \& Smith, A. N. (2016). Augmented reality: Designing immersive experiences that maximize consumer engagement. Business Horizons, 59(2), 149-161, doi: 10.1016/j.bushor.2015.10.003.

Stoyanova, J., Brito, P. Q., Georgieva, P., \& Milanova, M. (2015). Comparison of consumer purchase intention between interactive and Augmented Reality shopping platforms through statistical analyses. Innovations in Intelligent SysTems and Applications (INISTA), pp. 1-8. Madrid, Spain, 2-4 September 2015.

Tüchler, M., Schwarz, V., \& Huber, A. (2005). Accuracy of an UWB localization system based on a CMOS chip. 1st Ultra-Wideband Expert Talk (UET'05). 